\title{
Study on the Management System of Farmland Intelligent Irrigation
}

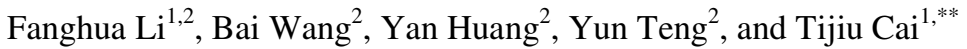 \\ ${ }^{1}$ Forestry College, Northeast Forestry University, Harbin Heilongiiang, P.R China \\ ${ }^{2}$ Heilongjiang Water Conservancy Institute, Harbin Heilongjiang, P.R China \\ fh63asohu.com, caitja263.net
}

\begin{abstract}
To achieve the unification of precise irrigation management, the system integrated GSM wireless communication technology, sensor technology, computer technology, automatic monitoring, and control technology in the study process. The system included the acquisition subsystem, the intelligent decision subsystem, and automatic control subsystem, with the advantages of the GSM internet, such as wide coverage area, powerful property of antiinterference, the remote data transmission was realized. The application of Java development platform and SQL Server 2000 achieved processing of real-time data. By testing and applicating, the system improved management level of agricultural water-saving irrigation for different accumulated temperature region, planting crops, soil type in Heilongjiang Province.
\end{abstract}

Keywords: Wireless data transmission, intelligent decision-making, irrigation automatic control, farmland moisture management.

\section{Introduction}

With the increasingly intensifying water resources scarcity and imbalance between supply and demand, countries actively explore effective water-saving ways and measures in the world [1]. Irrigation agriculture is one of the biggest water consumer in the world, the optimal management of agriculture irrigation can save large amount of water resources. The water resource of China is very deficient and unevenly distributed, which is especially more severe in north regions, therefore, it is imperative to develop water-saving agriculture [2]. Intelligent decision support system used in agriculture started in mid and later period of 70's in the 20th century, it has become mature gradually after 30 years development[3]. Irrigation automatic control mode has many advantages such as saving-water, energy and labor saving and so on, also it can eliminate adverse effects caused by human factor in the process of irrigation, improve the accuracy of operation, it is beneficial to managing scientific the irrigation process and extending the advanced technologies. The exploration and

\footnotetext{
Foundation project : national science and technology support project (2007BAD88B01).

*** Corresponding author.
} 
application of irrigation decision support system and water-saving irrigation professor system not only can provide scientific decision foundation and decision scheme for scientific management and optimal allocation of water resource, improve water utilization rate, dull the supply and demand contradiction of water resource, lighten the peasants' work intensity ${ }^{[4]}$, but also promote the development of agricultural information and science technology industry, it is very important for the realization of water-saving irrigation automation.

Agricultural intelligent irrigation management system is accurate irrigation technology, which synthesizes the science and technology achievement of modern agriculture irrigation, establishes the water utilization management measures of agriculture irrigation, and realizes the irrigation regulate operation as the center. The system integrates GSM ( Global System for Mobile Communication) network wireless communication technology, sensor technology, computer technology, and agriculture irrigation automatic control technology, carries on calculation, analysis and decision-making based on collected information such as soil moisture, crop drought, and meteorology factors and so on, makes out irrigation forecast and decision, determines the time and amount of water irrigation, uses the decision results to automatically control and monitor the irrigation equipments simply and rapidly, carries out timely and suitable dynamic management of intelligent automatic irrigation for the field-crops, sets up the precision amount control field intelligent irrigation management system with remote monitoring, wireless transmission, rapid diagnosis, intelligent decision, and precision control functions, realizes modernization and automation of the irrigation water management means.

\section{The Basic Structure and Characteristic of the System}

Field intelligent irrigation management system is divided into central monitor layer and substation monitor layer according to control grade. Central monitor layer timely monitors the field situation of each area, directly sends out instructions to control executive element work by controller, plays a general monitor role, the system is managed by the central monitor layer. Substation monitor layer is placed at the controlled field, each substation has independent control system, responsible for executing the commands of central monitor layer and users, and the control of the equipments and the management of information of the station. Central monitor layer and substation monitor layer are composed of acquisition subsystem, intelligent decision subsystem, and automatic control subsystem. The system collects the information by monitoring acquisition subsystem, the information through GSM network transfers timely or real-time to the monitor central host according to users' requirements. The monitor central host connects with the computer, which makes scientific irrigation task list by intelligent decision system, the control center host gives out irrigation commands through GSM network, automatic control subsystem automatically performs the irrigation work according to the instruction of decision support system. 


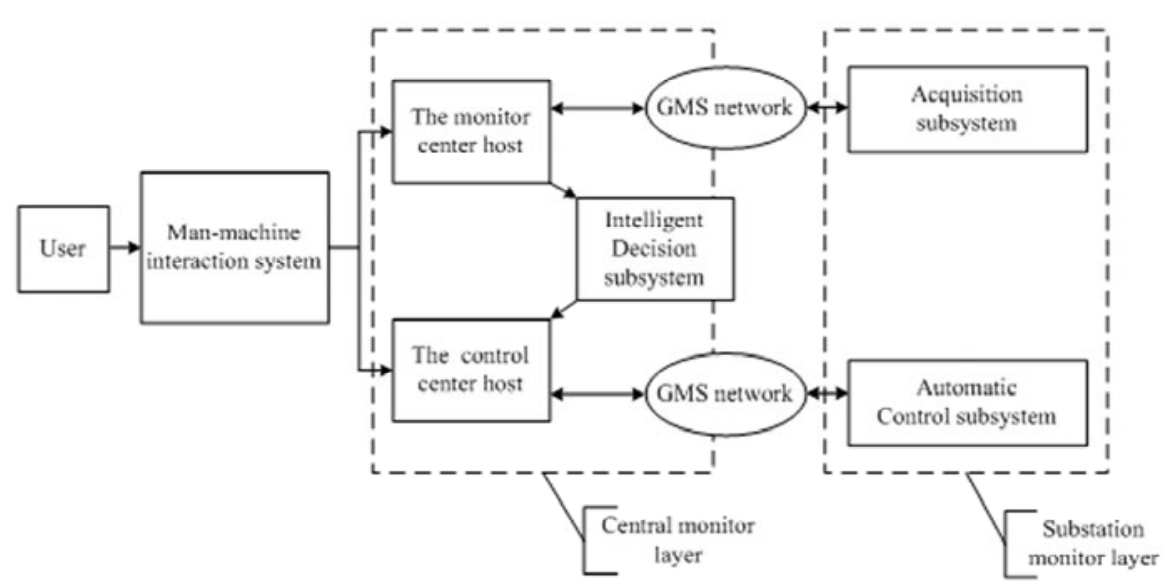

Fig. 1. The basic structure of field intelligent irrigation management system

\subsection{Acquisition Subsystem}

\subsubsection{The Basic Function of the System}

Acquisition subsystem collects the basic information of the field by all kinds of equipments, the basic information such as soil water, atmospheric temperature, atmospheric humidity, the flow of water meter in flooding and so on. Acquisition system has the GSM network as the basic support for data transmission, has the Short Message Service short message as communication carrier ${ }^{[5][6][7]}$, realizes the remote wireless bidirectional data communication from one point to multi-point. Data acquisition and transmission are the basic link of the whole system decision and control, the data acquisition and transmission, which has a lot of characteristics such as the low operation cost due to low on short messages cost, the stable reliability, the higher rate of data transmission, low error code rate and so on, perform timely and real-time data acquisition, stresses the timeliness and dynamic of data monitor, provides accurate and timely information guarantee for realizing water monitor and decision of large-area field.

\subsubsection{Working Principle of the System}

According to the requirement of area characteristic and acquisition factors, by the operation on the interface between the man and the computer, user transfers the instruction to the host in the acquisition monitor center to acquire indexes through COM port, the host of acquisition monitor center uses the GSM wireless communication equipments, sends the setting command to the terminal of data acquisition in the form of short message to go on initialization. According to the requirement of received acquisition instruction, acquisition monitor substation collects the information from the soil water sensor, temperature sensor, humidity sensor and flow sensor in the field. Monitor substation based on solar energy battery can realize timely and real-time reception and transmission of information collected, which is highly effective. Monitor substation sends the information collected to the host of acquisition monitor center in the form of short message, users can master best 


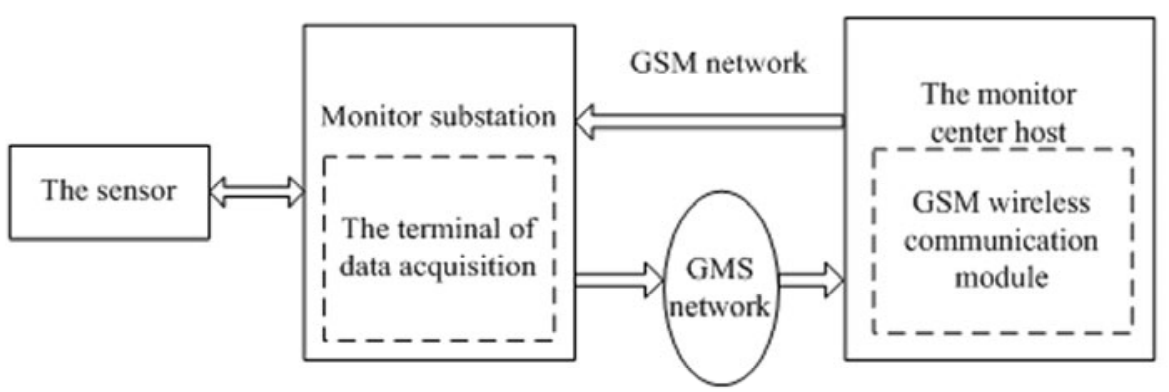

Fig. 2. Work principle flowchart of data acquisition system

and comprehensive data information of field water moisture and meteorological factors by means of the fixity of timing acquisition and the random of real-time acquisition, then stores the data to database. The work principle is shown as in Fig.2 .

\subsection{Intelligent Decision Subsystem}

\subsubsection{Basic Function of System}

Intelligent decision subsystem is a total integration system, which uses database, people and computer interaction to go on multi-mode organic combination, it assists decision-maker to realize scientific decision[8]. Intelligent decision system with the meteorological data and water moisture data as the basis, using the water balance model, combines with the theoretical basis and characteristic of crop water requirement in the system knowledge base, soil properties and irrigation methods, makes reasoning computation according to some rules, makes out water saving irrigation decision for the crop planted in one area or one field, there are some irrigation reference factors, that is, making sure the crop need to irrigate or not, and when it needs to irrigate, comprehensive soil water content can reach the lower limit index suitable for crop growing or not, weather forecast and the growing stage of the crop is in the stage suitable for regulated deficit irrigation or not. It takes protective irrigation alarming mode as soil moisture content diagnose system, timely makes out accurate irrigation decision, sends out concrete operation instruction to control subsystem through the interface of interaction between man and computer.

\subsubsection{Work Principle of the System}

Field monitor substation sends acquisition information collected by soil water sensor, temperature sensor, humidity sensor and flow sensor through GSM network to the host of monitor center, decision system takes database to receive collected information as the basis, has mode base as the support of system calculation and statistics, has the method base as the theoretical guidance of mode base calculation and statistics, has the knowledge base as the support for system theory and experience operation, makes calculation, statistics, analysis of the information, then makes out irrigation decision, makes effective and water-saving irrigation plans, realizes scientific guidance to area irrigation. The work principle is shown as in Fig.3. 


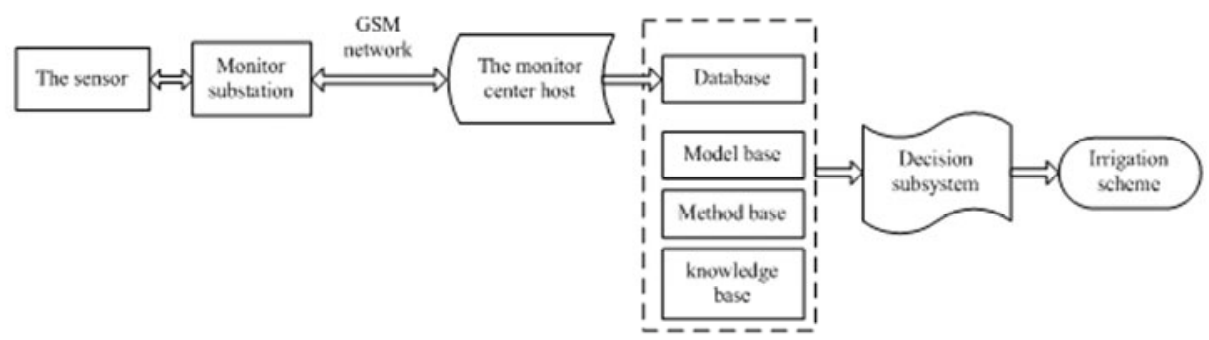

Fig. 3. Work principle flowchart of intelligent decision subsystem

\subsection{Automatic Control Subsystem}

\subsubsection{Basic Function of the System}

Field intelligent irrigation automatic control subsystem realizes scale and automation of production, raises labor productivity[9]. Control subsystem is the remote automatic irrigation monitor system of GSM wireless communication, it transmits irrigation decision scheme instruction of intelligent decision subsystem, via the host of system control to control substations by GSM network. Control substations guide the irrigation controller to open or close according to the received irrigation decision instruction and transmit irrigation water parameters collected to computer via the host of control centre, in order to achieve the aim of wireless automatic irrigation.

\subsubsection{Work Principle of the System}

Field monitor substation sends information collected by soil water sensor, temperature sensor, humidity sensor and flow sensor by GSM network to the host of monitor center. According to mode base, method base, knowledge base and database, decision system makes out irrigation decision. Control subsystem sends irrigation decisions via GSM network to field control substation by the host of control center. Control station realizes precision irrigation control according to single control electromagnetic valve's open or close for irrigation decision task. The work principle is shown as in Fig.4.

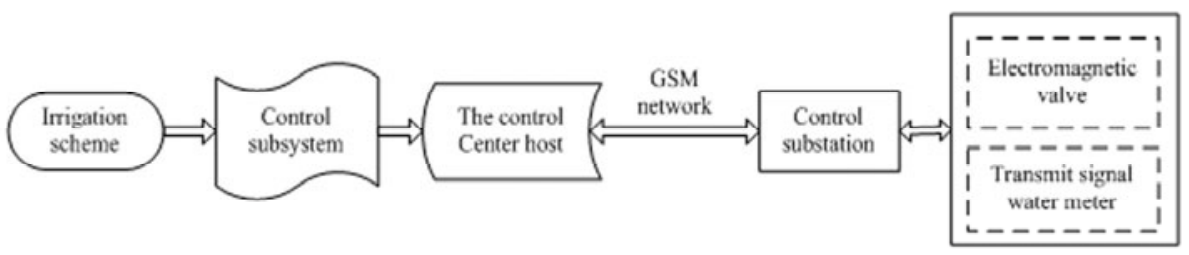

Fig. 4. Work principle flowchart of automatic control subsystem 


\section{Database Design}

Database is a data storage space for storing irrigation management decision and model operation, an application software integral with the unified management of data which is massive, complex in structure, abiding and sharing as goal, meanwhile it is independent, comprehensive, universal, sharing and easy in maintenance [4][10]. The level of database structure design directly effected both the efficiency of the system and the effects of implementation[11]. Database design includes basic database, real-time database and spatial database design, it is mainly composed of graphic library, all kinds of acquisition information and attribute information of all kinds of ground objects [12][13]. In the field intelligent irrigation management system, Database Management System is used mainly to manage and maintain the data in database, makes the information storage and reading systematic, standardized and automatic, including browsing, query, renewal, adding, backup and recovery of data and so on.

Database of system used the efficient relational database to combine with Windows NT/2000 and windows 9x,the characteristics of operating system were adequately used. Combinning with GSM wireless communication technology, the management system of farmland intelligent irrigation was developed by the test function and the graphic user interface of Java virtual platform, which was based on virtual instrument technology. Established database includes six functions as follows:

(1) Meteorology database. It can store, query and modify the basic information of meteorology factors, such as accumulated temperature, rainfall, atmospheric temperature and relative humidity etc.

(2) Crop information database. It can store, query and modify the basic information of crop growth and development, including crop growth stage, days of growth stage, the depth of effective water absorption layer of root in each growth stage, suitable water upper and lower limits in each growth stage etc.

(3) Soil information database. It can store the basic soil water dynamic information of management regions, including field capacity and bulk density (The type of soil is divided into black soil, chernozem, sandy loam soil, meadow soil and saline soil and so on ), and can query and modify the basic dynamic information of soil water.

(4) Irrigation regional database. It can store, query and modify the basic information of irrigation regions, including the crop, soil, equipment, picture and characters etc in the regions.

(5) Irrigation elements database. It can store, query and modify the basic information of the ways and quota of irrigation. The ways of irrigation include sprinkler irrigation, drip irrigation and furrow irrigation. The quota of irrigation includes the quota of different accumulated temperature zones, different crops and different growth stages.

(6) Irrigation alarming database. It includes irrigation time, irrigation quantity and alarm limit of soil water etc.

\section{Application and Analysis of the System}

The management system of farmland intelligent irrigation was tested and applied between the year 2009 and the year 2010 in some areas of Heilongjiang Province, 
such as Qinggang, Dumeng, Zhaodong, meanwhile, was improved and upgrade. The system used field sensors to achieve the collecting work, the measurement accuracy of soil water sensor reached $\pm 2 \%\left(\mathrm{~m}^{3} / \mathrm{m}^{3}\right)$ in $0-50 \%\left(\mathrm{~m}^{3} / \mathrm{m}^{3}\right.$ reached stable after electrifying. The solar cell of power supply system had some advantages to avoid hindering collection of information with insufficient electricity under rainy weather condition, the system could run normally, such as non equalization charging, small self-discharge, without the leakage of liquid, without overflow of acid gas. By processing the information, acquisition monitor substation timely or real-time transmitted digital signal to the host of acquisition monitor center according to users' requirements. Acquisition monitor substation contained acquisition circuit, gate circuit, ATM processor, cpu acquired soil water by sensor, transfered the analog into digital information, the remote transmission of signal to the host of acquisition monitor center could be achieved by GSM wireless module. The host of acquisition monitor center was composed of programmable array multiplexer, constant current source, isolation transmit amplifier, 12 bit A/D converter, using 32 bitARM processor, operating system was MC/OS-II, storage capacity was $2 \mathrm{M}$ byte. The host of acquisition monitor center received the GSM information from acquisition monitor substation and stored monitoring data for 9 months. The host of acquisition monitor center connected with computer, the computer intelligent irrigation management software calibrated the information of database with the support of mode base, methods base, knowledge base, the processing physical quantities were displayed software interface, processing results were stored the relative database, according to the soil moisture and growth condition of plant, decision system gave out the irrigation time, irrigation water, and other relevant information after analyzing and calculating, then, sent irrigation command to the host of control center. The host of control center was composed of programmable array multiplexer, constant current source, isolation transmit amplifier, using 32 bit ARM processor, operating system was MC/OS-II. The control substation not only were acquisition terminal but also had irrigation control performance, it was composed of GSM communication module, data acquisition module, control module, power module. The control substation received the irrigation command from the host of control center, achieved irrigation tasks with irrigation controller, then, sent executed information of irrigation tasks to the host of control center.

Field intelligent irrigation management system sets up design features with area characteristics, besides some functions such as irrigation decision support system, water-saving irrigation expert system and artificial intelligence irrigation management system.

(1) The system has different accumulated temperature zones, different crops and different soil types in Heilongjiang province as the basic indexes, establishes the comprehensive database combined with crop growth information and soil information essential for field irrigation, satisfies actual demand for irrigation decision and guidance under the condition of different planting structures and different characteristic regions, and also can modify, newly add parameters to the database and has better expansibility.

(2) The system has the GSM network and solar energy component power supply system as support, realizes timing or real-time reception and remote wireless 
transmission of various acquisition information in field. Real-time monitor of data information provides powerful guarantee for irrigation decision.

(3) The system establishes water diagnosing index system which is based on field moisture content of crop physiological characteristics and soil physical characteristics, monitored by sensor, using man and computer interaction system design, The system performs the alarming function to soil water deficiency or sufficiency, implements scientific irrigation management and system monitor maintenance.

(4) The system regional management design in different layers overcomes inhomogeneity in spatial distribution on small scale, implements dynamic monitor of point to point moisture content in field divisions, satisfies the hierarchy of time distribution on large scale, realizes concentration and integration of automatic irrigation in management regions, sets up precision and effective monitor and irrigation management technique modes which satisfy regional characteristics.

\section{Conclusions}

Field intelligent irrigation system is prepared mainly on the basis of aggregating large amount of knowledge information, in connection with characteristics of the soil properties of main crops, the law of crop water requirements, and crop evapotranspiration etc a series of data indexes of soybean, sunflower and sugarbeet, etc, planted in different accumulated temperature zones in Heilongjiang province. With the support of computer, the system uses remote monitoring, realizes the monitor of soil moisture and timely monitors the amount of water consumption and requirement in field crop growth period, gives accurately values of crop water requirement, makes out irrigation decisions, and the irrigation control system carries out automatic irrigation according to decision content, which makes dispersed agriculture facilities and management areas a whole body, improves the operation efficiency of agriculture, reduces the cost of production, provides proper moisture growth environment for crops, realizes the objects of efficiency, water saving and yield increase.

\section{References}

1. Ma, J.: The Study of Accurate Irrigation and Fertilization Automatic Control System. Shanxi Agricultural University. Master Degree Dissertation, p. 5 (2005)

2. Yang, J.: The Application of Software Engineering in the Development of Agricultural Expert System. Journal of Agricultural Mechanization Research 3, 224 (2005)

3. Deng, J.: The Design and Implementation of Intelligent Decision System for Apply Fertilizer and Irrigation. Central China Normal University. Doctorate Dissertation, p. 2 (2006)

4. Cui, J., Ma, F., Zheng, Z., et al.: Study on Field Water Irrigation Management and Automatic Control System Based on GSM. Water-saving Irrigation 5, 30-31 (2005)

5. Zhang, Z., Zhao, P., Yang, Z., et al.: System of the Hydrology Information Remote Collection Based on GIS and GSM. Journal of Anhui Agricultural Science 36, 6585-6586 (2008) 
6. Shu, Y.: Real Time Collection System of Soil Moisture Based on Short Message. Guizhou University. Master Degree Dissertation, pp. 6-7 (2006)

7. Li, N., Liu, C., Li, Y., et al.: Development of remote monitoring system for soil moisture based on $3 \mathrm{~S}$ technology alliance. Transactions of the Chinese Society of Agricultural Engineering 26, 169-172 (2010)

8. Zhang, W., Liu, X., Gu, H.: Development of Real-time Irrigation Decision Support System Software of Irrigation Regional. Yellow River 29, 54 (2007)

9. Hao, W., Peng, X., Geng, Q., et al.: Intelligent Irrigation Control System Based on ARM. China Rural Water and Hydropower 5, 24 (2006)

10. Shang, H., Wang, Z., Chai, P.: Research and Development of Water-saving Irrigation Database and Its Management System. Research of Soil and Water Conservation 9, 97-98 (2002)

11. Yang, J.: Research on Decision Making Support System for Precise Irrigation of Wheat in the Hexi Oasis Irrigation Areas. Gansu Agricultural University. Master Degree Dissertation, pp. 42-43 (2007)

12. Sun, M., Cai, D.: Research on Irrigation Areas Water Resource Management Based on Ground Information System. Water Saving Irrigation 1, 43 (2007)

13. Chen, L., Huang, J.: Integration of Irrigation Management Model and GIS and Its Application. Journal of Irrigation and Drainage 3, 29-30 (2003) 